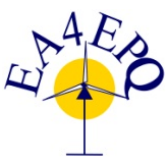

International Conference on Renewable Energies and Power Quality (ICREPQ'13)

Bilbao (Spain), 20 ${ }^{\text {th }}$ to $22^{\text {th }}$ March, 2013

Renewable Energy and Pourer Quality. Tournal (RE\&PQJ)

ISSN 2172-038 X, No.11, March 2013

\title{
Energy Storage Requirements to match Wind Generation and Demand applied to the UK network
}

\author{
W.-G. Früh \\ Institute of Mechanical, Process and Energy Engineering, School of Physical Sciences and Engineering, \\ Heriot-Watt University, Riccarton, Edinburgh EH14 4AS (UK) \\ E-mail: w.g.fruh@hw.ac.uk
}

\begin{abstract}
Increasing the contribution from Renewable Energy Systems to the electricity network places increasing demands on both, power quality assurance as well as energy storage or back-up generation availability.
\end{abstract}

Based on a set of forty typical UK years, generated by matching 10 years of hourly wind speed observations at a typical site in Scotland to four years of hourly UK demand data from the National Grid, the statistics of the hour-byhour matching of wind generation to the demand and the cumulative effects of electricity surplus or deficit is analysed to obtain measures for the energy requirements and their associated time scales to complement the wind power through energy storage technologies or complementary scheduled generation.

The findings for the UK case suggest that the overwhelming majority of energy balancing occurs over a time scale of less than a week, where the energy storage capacity is around $10 \mathrm{MWh}$ per $\mathrm{MW}$ of installed wind capacity.

\section{Key words}

Wind resource, Wind integration, Energy storage, Demand matching.

\section{Introduction}

Wind power generation is one of the fastest growing industries in the developed world, with an installed capacity of $194 \mathrm{GW}$ in 2010 through large wind farms, projected to grow by $15-23 \%$ per year over the next 5 years [1]. Considering that some of the wind farms now reach an installed capacity in the GW range, even a small reduction in output can amount to a significant change in the power feed into the grid which must then find other sources of electricity to balance a shortfall, or reduce other generation to balance a surge in wind power. This could be achieved by curtailing scheduled generation, which comes at a cost of operating thermal plant well below their best efficiency. Alternatively, energy storage technologies such as pumped storage hydropower [2], Compressed Air Energy storage [3] or batteries [4], can be used to balance the system at 'energy' time scales of several minutes to months [5].

The aims of this study are to provide a detailed and systematic analysis of a realistic wind resource in the context of a realistic demand scenario. For this, a 10year long record of wind speed data from a UK Met.Office surface station [6] was combined with a 4year record of national electricity demand data from the UK's National Grid [7]. These were then combined to forty sample years by matching each wind power year to each demand year.

\section{Methodology}

The wind speed data were taken from the UK Met. Office's surface station Machrihanish at the west coast of Scotland from the MIDAS Land Surface Stations database provided by the British Atmospheric Data Centre [6] covering the period from 1 January 2000 to 31 December 2009. The data are supplied in a time series of 

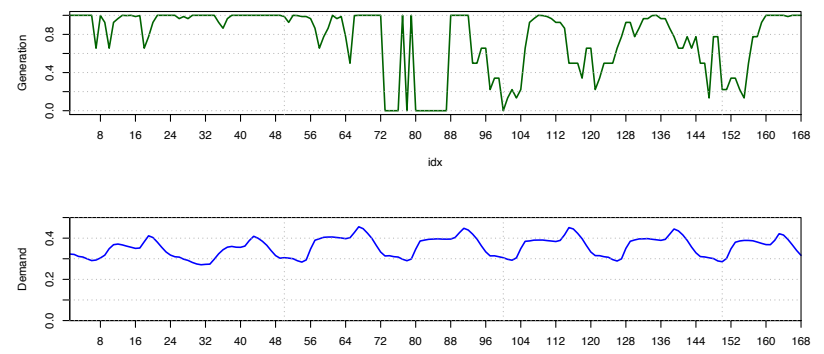

idx

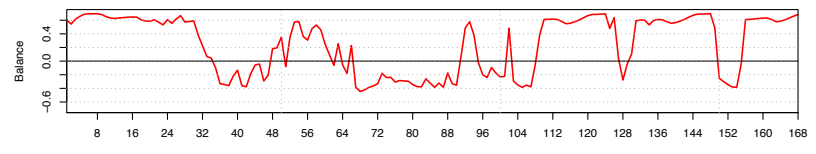

time (h)

Figure 1. Sample time series covering a week of (top) wind power, (middle) demand, and (bottom) the balance given by the difference between generation and consumption.

hourly weather data including the hourly mean wind speed in knots to the nearest knot $(1 \mathrm{kn}=0.5144 \mathrm{~m} / \mathrm{s})$. The anemometer at this site is placed $10 \mathrm{~m}$ above ground and well exposed to the Atlantic. As such, it represents a site with an outstanding wind resource but it has been shown to be highly correlated with inland stations in the same latitude belt of central Scotland [8]. For that reason, it is believed to be a good representative of wind farms in Scotland. The wind speeds were then scaled up by a logarithmic velocity profile as

$$
u(z)=u_{10} \frac{\ln \left(z / z_{0}\right)}{\ln \left(10 \mathrm{~m} / z_{0}\right)}
$$

These scaled up wind speeds were then feed through a generic wind turbine performance curve with cut-in wind speed of $4 \mathrm{~m} / \mathrm{s}$, rated wind speed of $13 \mathrm{~m} / \mathrm{s}$ and cut-out wind speed of $25 \mathrm{~m} / \mathrm{s}$ with unit rated power. To represent a typical performance of actual wind farms in the UK with a capacity factor of around $30 \%$, the wind speeds were scaled up to a low hub height of $30 \mathrm{~m}$ and a low surface roughness of $10 \mathrm{~mm}$. This resulted in a decadal mean capacity factor of $C_{C}=30.7 \%$, with a minimum of $25.7 \%$ and a maximum of $32.7 \%$.

The demand was taken from the National Grid's published half-hourly total demand data [7], which was then reduced to a mean hourly demand by averaging over on-the-hour demand and the following half-past demand for the years from 1 January 2008 to 31 December 2011. The individual years had a mean demand of $39.1 \mathrm{GW}$, $37.6 \mathrm{GW}, 38.3 \mathrm{GW}$, and $36.5 \mathrm{GW}$, respectively, with an overall 4-year mean of $D^{*}=37.9 \mathrm{GW}$. As can be seen, there is no systematic change in the mean demand over the four years, and each can be taken as a representative of typical current demand. To generate a range of typical wind power - demand years, each of the ten wind power years was matched to each of the four demand years, giving a total of forty typical years.

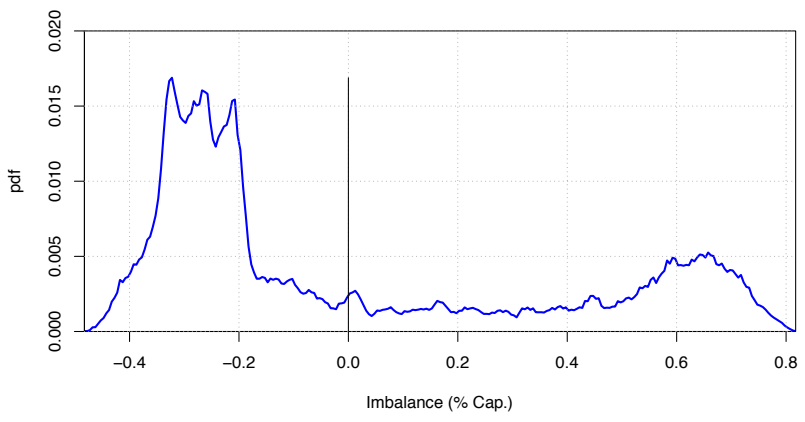

Figure 2. Distribution of the instantaneous imbalance against the value of the imbalance.

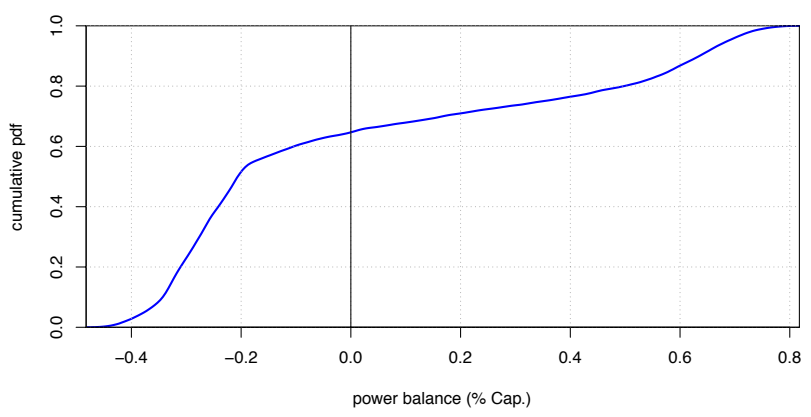

Figure 3. Cumulative distribution of the power balance.

For the analysis, the premise was that the wind power should contribute to the total consumption the fraction it can meet based on the long-term average capacity factor. Therefore, the fraction of the demand expected to be met by wind is $D=C_{C} / D^{*}$. Then the assumption was that if the instantaneous (hourly) wind power, $P$, was equal to that fraction at that time, the system was in balance but if $P>D$, then there would be a surplus which could, for example, be used to charge an energy storage facility, and if $P<D$, then there would be a deficit which would be complemented by the use of the energy storage facility. A week's example of the wind power generation, fraction of demand to be met, and the resulting balance is shown in three graphs in Figure 1, respectively.

For the purpose of this analysis, it was assumed that all surplus would be accumulated in energy storage and all deficit met from that storage, where the transfer of electricity to and from the storage was without losses. In this case, the energy level in the storage facility after a given time point is equal to the sum of the level at the previous time point and the balance during that current time interval. If each of the sample years starts with a zero reference level, the storage level is the cumulative sum of the balance. Since power is scaled to a unit rated power and the data are hourly data, the energy level in the storage is in units of $\mathrm{MWh}$ per MW installed wind power. 


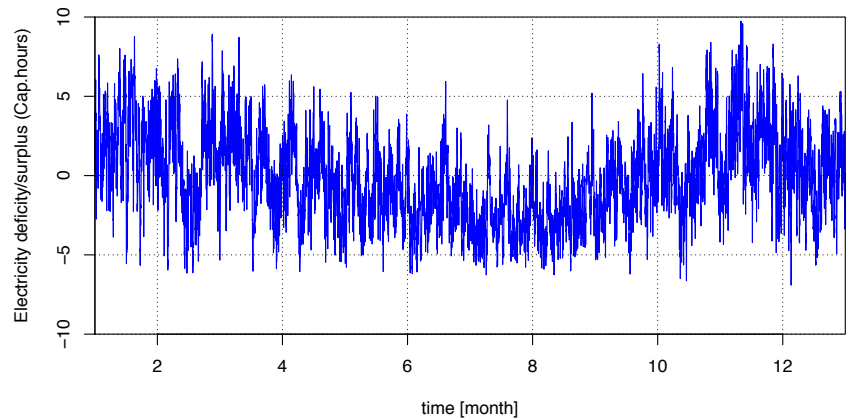

Figure 4. Cumulative sum of balance covering one of the sample years (wind year 2010 and demand year 2009).

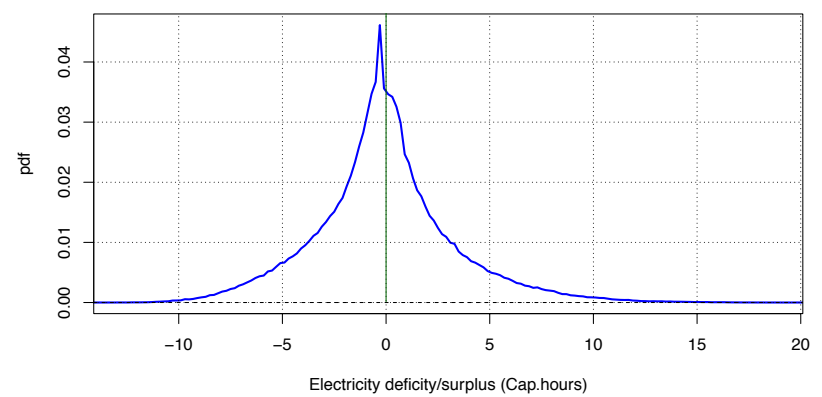

Figure 5. Distribution of cumulative balance covering all forty years.

\section{Results}

Figure 2 shows the distribution of the hourly imbalance as the probability density function against the magnitude of the imbalance scaled by the installed capacity. This shows a highly skewed distribution with the majority of shortfall in the range of 20 to $40 \%$ of the installed capacity against a much broader range of surplus generation with a moderate peak around $60 \%$ of the installed capacity. This can be explained by the fact that the mean capacity factor is $30 \%$. So, for a typical demand level of $30 \%$ of the installed wind power, the shortfall is only $30 \%$ if the turbine is operating whereas the surplus would be $70 \%$ if at that typical demand level the turbine were to operate at its rated power. Figure 3 shows the cumulative distribution of this power balance and shows that the wind power is less than sufficient for around $66 \%$ of the time despite matching the demand fraction to the generated wind power in the long term. This figure also shows that an available reserve generation of $20 \%$ of the installed wind capacity would only be sufficient to cover a further $15 \%$ of the time while a reserve generation capacity of $40 \%$ of the wind capacity would be sufficient to meet the electricity demand for $97 \%$ of the time.

As the bottom panel of Figure 1 shows, the imbalance is a highly volatile function, largely due to the wind variability exacerbated by the turbine's nonlinear performance curve. Since the focus of this paper is energy storage rather than power quality, we concentrate on the cumulative sum of the power balance. Figure 4 shows the cumulative balance - or storage level in the energy storage facility - for one of the sample years. Three key features emerge from this

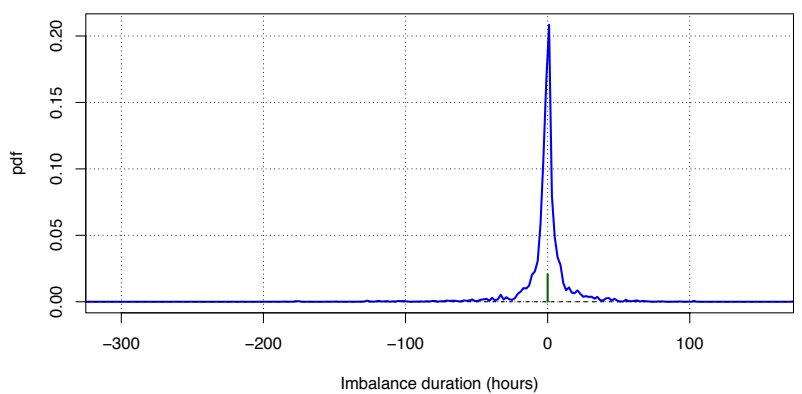

Figure 6. Distribution of duration of episodes of electricity deficit (negative periods) and electricity surplus (positive periods).

graph: still a very high volatility even of this integrated quantity, a clear seasonal cycle with a predominance of energy surplus in the winter months and deficit in the summer months, and a few isolated periods of deficit in February, October and December. Figure 5 shows the distribution of the imbalance which shows a sharp peak just below the balanced point and a sharp drop-off to electricity deficits which extend to about $-10 \mathrm{MWh}$ per MW of installed wind capacity mirrored by a slightly less sharp decay to electricity surplus reaching a little beyond $+10 \mathrm{MWh}$ per MW. This distribution is reminiscent of a Levy distribution which is common for event driven processes found in, for example, geophysical time series [9].

For electricity storage, it is not only the amount of energy which is importance but also the duration over which this must be stored. This quantity is shown in Figure 6, with periods of energy deficit as negative durations and periods of energy deficit as positive durations, which demonstrates that most of the time the duration of each period of imbalance is very short, with very few longer periods. The longer periods are highly asymmetric, with periods of energy deficit extending to 325 hours, while those of surplus have a maximum of 174 hours. Showing this as the cumulative distribution and only showing the range up to 48 hours of deficit or surplus in Figure 7, shows that most of the energy surplus or deficits are balanced again after 12 hours and that $99 \%$ of the durations of surplus last less than two days, while only $98 \%$ of the durations of deficit last less than two days.

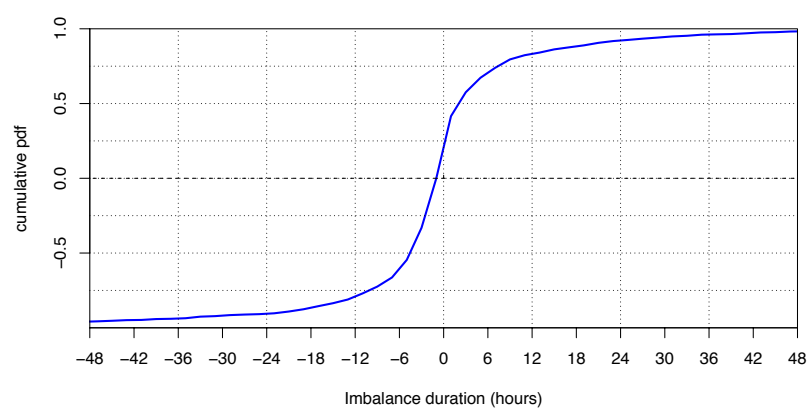

Figure 7. Cumulative distribution of durations of episodes of deficit and surplus electricity. 


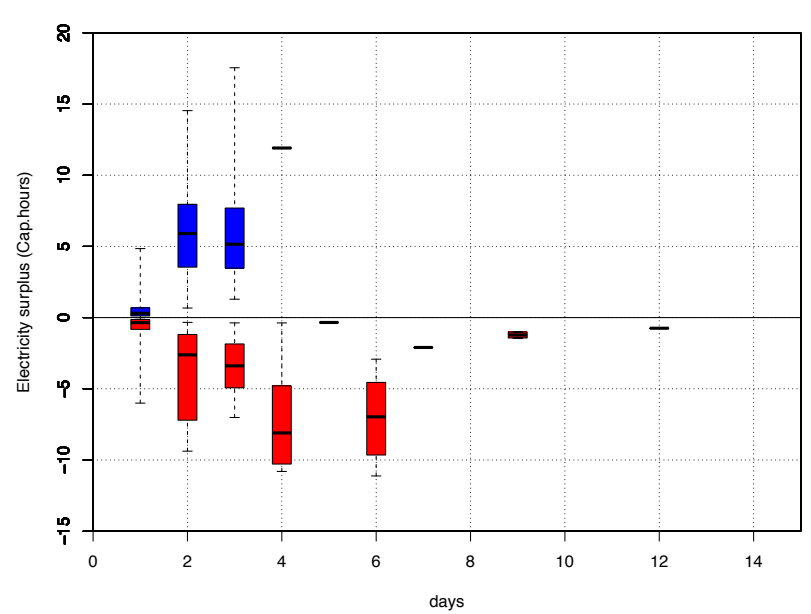

Figure 8. Box plot of electricity surplus (positive blue) and electricity deficit (negative red).

The fact that the periods of imbalance can be divided into within-day and 'over a few days' can be used to combine durations of imbalance into 24-hour bands and to display the duration and magnitude of energy surplus or density as a box-and-whisker plot in Figure 8 which shows the quartiles of the energy surplus/deficit magnitude distribution for each 24-hour duration band. This shows that the within-day imbalances tend to be very small: three-quarters of the time much less than $1 \mathrm{MWh}$ per MW installed up to a maximum of around $5 \mathrm{MWh}$ per MW. As Figure 7 showed, these periods make up the vast majority of imbalances. However, a few periods of surplus extend over two to three days, with a few isolated events extending over four days. The situation is somewhat but crucially different, were several events extend to 6 days and a few isolated events lasted up to 12 days. It appears that the very long events of deficit only require a storage volume of around $2 \mathrm{MWh}$ per $\mathrm{MW}$ installed wind capacity, whereas the $4-6$ day periods of deficit required up to $10 \mathrm{MWh}$ per $\mathrm{MW}$, and some of the surplus periods requiring up to $18 \mathrm{MWh}$ per $\mathrm{MW}$ of installed wind capacity.

\section{Discussion and Conclusions}

We analysed the required stand-by generation capacity and energy storage to match electricity demand. To quantify this balance, the premise was that the wind power at any time should match a fraction of the demand equal to that which the long-term mean wind power could contribute to the long-term mean demand. Any excess would be added to energy storage and any deficit met by that energy storage without losses. The analysis was based on matching ten years of wind data from a site in Scotland and four years of demand data from the UK's National Grid. The wind data were rescaled to a capacity factor of $30 \%$ which is typical for the UK as a whole, and the demand data were scaled so that its long term mean matched the long-term mean capacity factor.
In the context of the capacity factor of $30 \%$, a pronounced asymmetry between insufficient and excess generation was observed, where the majority of the insufficient generation was between 20 and $40 \%$ of the installed wind capacity distributed evenly either side of the capacity factor value. The excess generation, however, extended to $80 \%$ of the installed wind capacity. The power deficit in excess of the mean capacity factor occurs in calm weather during peak demand while the most substantial excess generation occurs during windy off-peak times. Due to the asymmetry of the distribution, wind power would match the demand only $34 \%$ of the time. To guarantee power supply at the expected mean wind power for $97 \%$ of the time, the stand-by generation must be $40 \%$ of the installed capacity which is a third above the mean power.

The amount of electricity required to be stored to make up a later shortfall was still found to be very volatile, with a surplus exhausted and replenished mostly over time scales of 12 hours, in line with the demand pattern of 12 hours low off-peak demand and 12 hours higher day-time demand. However, a small number of longer periods of surplus or deficit was found, some of which aligned with the seasonal cycle - more surplus in winter and more deficit in summer - but also with individual typical weather types which often occur in mid to late February and mid- to late-October. These periods can be associated with high-pressure blocking events at a time of seasonally induced higher typical demand levels. While these events might be rare, with only one or two events in a year leading to a persistent electricity deficit on time scales of 7 to 12 days, they are critical times for network operators as well as for the electricity trading market. Ensuring stable electricity supply during one or two of these events in a year may contribute to about half of the annual costs of providing sufficient alternative generation. This is reflected in rapid and extreme changes of the electricity spot market price [10], for example a rapid rise from a typical level of around $€ 40$ per MWh to $€ 360$ per MWh in the Central Western European electricity wholesale market during a blocking event in February 2012 [11], when large areas of Europe were experiencing a period of cold and calm weather.

Overall, the energy storage capacity to deal with the majority of within-day supply-demand shifting is of a moderate magnitude, around $1 \mathrm{MWh}$ per MW installed. The longest period place a somewhat larger demand of around twice that energy storage. The largest stress on the system is placed by the $9 \%$ of events lasting between 2 and 6 days, with a need to absorb occasionally up to $18 \mathrm{MWh}$ excess electricity per MW installed, and to ensure that up to $10 \mathrm{MWh}$ per MW installed are available at the beginning of the most severe period of wind power deficit.

The results are very much dependent on the local context of the UK demand and wind resource with their seasonal cycle generally well aligned. Other sites, for example central and southern Europe or continental climates in general may show different seasonal patterns, and so may the demand. For example, southern countries are likely 
to have high demand in the summer months when air conditioning is used, as well as higher demand from the tourist industry in general.

Translating this into the UK context of a mean demand of around $40 \mathrm{GW}$, a maximum peak demand of around $60 \mathrm{GW}$ and a typical wind capacity factor of $30 \%$, we would require an installed wind capacity of around $130 \mathrm{GW}$ complemented by a back-up supply of $160 \mathrm{GW}$ with a storage capacity of up to around $2000 \mathrm{GWh}$ if we wanted to come all electricity from wind alone. Furthermore, the results have to be interpreted in the light of the assumption that all excess electricity is stored and all deficit is compensated by the stored electricity without any losses. However, the analysis is easily adapted to realistic storage technology or facilities by dividing the storage requirements by the round-trip efficiency. Clearly, the choice to supply a whole nation's electricity by a single and highly variable renewable resource alone is extreme and unrealistic. However, this approach now be developed further to a model which combines renewable resources, storage facilities (with realistic round-trip efficiencies), scheduled generation to meet expected demand, and responsive 'backup' generation to identify, for example, which blend of generation and storage technology provides the most reliable electricity supply at the lowest carbon cost.

A useful next step in the analysis is to blend the storage strategy with optimum scheduled generation using alternative sources. Some of the electricity shortfall would be more effectively met by good forward planning of scheduled generation to optimise their efficiency and productivity while reducing the electricity storage requirements. If it is possible to forecast extended periods of additional need, and to combine the knowledge of the daily cycle and $48 \mathrm{~h}$ weather forecasts, it will be possible find optimum solutions to the problem in which the demand and availability of stand-by or traditional generation as well as the use of wind power are maximised.

\section{Acknowledgements}

We would like to thank the UK Meteorological office for providing access to the wind data from the MIDAS record through the British Atmospheric Data Centre (www.badc.ac.uk) and for providing additional anemometer details.

\section{References}

[1] GWEC. Global wind statistics 2010. Global Wind Energy Council, 2011.

[2] C. Bueno and J.A. Carta. Wind powered pumped hydro storage systems, a means of increasing the penetration of renewable energy in the Canary Islands. Renewable and Sustainable Energy Reviews 10:312-340, 2006

[3] D. Zafirakis and J.K. Kaldellis. Autonomous dualmode CAES systems for maximum wind energy contribution in remote island networks. Energy Conversion and Management 51:2150-2161, 2010.

[4] J. Druitt and W.-G. Früh. Simulation of Demand Management and Grid Balancing with Electric Vehicles. Journal of Power Sources 216: 104 - 116, 2012.

[5] S. Vazquez, S.M. Lukic, E Galvan, L.G. Franquelo and J.M. Carrasco. Energy Storage Systems for Transport and Grid Applications. IEEE Transactions on Industrial Electronics 57: 3881-3895, 2010.

[6] UK Meteorological Office. MIDAS Land Surface Stations data (1853-current). NCAS British Atmospheric Data Centre. Available from http://badc.nerc.ac.uk/view/badc.nerc.ac.uk_ATOM _dataent_ukmo-midas; 2011.

[7] National Grid, Operating the electricity transmission networks in 2020, 2010.

[8] W.-G. Früh. Long-term wind resource and uncertainty estimation using wind records from Scotland as example. Renewable Energy 50:10141026, 2013.

[9] A. Witt and B. Malamud. Quantification of LongRange Persistence in Geophysical Time Series: Conventional and Benchmark-Based Improvement Techniques. Surveys in Geophysics, in press, 2013.

[10] E. Lindström and F. Regland, Modeling extreme dependence between European electricity markets. Energy Economics 34: 899 - 904, 2012.

[11] European Commission. Quarterly Report on European Electricity Markets. DG Energy Market Observatory for Energy 5(1), January - March 2012. 